\title{
Metabolic and Molecular Regulation of Dietary Polyunsaturated Fatty Acids on Prostate Cancer
}

\author{
Heng Zhao ${ }^{1}$, Beth R. Pflug ${ }^{2}$, Xianyin Laii, ${ }^{1,3}$ Mu Wang ${ }^{1 *}$ \\ ${ }^{1}$ Department of Biochemistry and Molecular Biology, Indiana University School of \\ Medicine, Indianapolis, IN \\ ${ }^{2}$ Department of Medicine, Division of Clinical Pharmacology, Indiana University School \\ of Medicine, Indianapolis, IN \\ ${ }^{3}$ Department of Cellular \& Integrative Physiology, Indiana University School of Medicine, \\ Indianapolis, IN
}

Running title: Quantitative proteomic analysis of prostate cancer cells treated with polyunsaturated fatty acids

Correspondence: Mu Wang, Ph.D.

Department of Biochemistry and Molecular Biology

Indiana University School of Medicine

635 Barnhill Dr., MS 4053

Indianapolis, IN 46202

USA

Phone: 001-317-278-0296

Fax: 001-317-274-4686

E-mail: muwang@iu.edu 


\title{
Keywords:
}

Prostate Cancer / Mass spectrometry / Fish oil / Multiple reaction monitoring (MRM) / Biomarker

\author{
Abbreviations: \\ $\mathbf{A R}$, androgen receptor; AUC, area under the curve; CE, collision energy; CM, \\ conditioned media; COX2, cyclooxygenase 2; DHA, docosahexaenoic acid; DP, \\ declustering potential; EPA, eicosapentaenoic acid; FA, fatty acid; FASN, fatty acid \\ synthase; FO, fish oil; LC-MS, liquid chromatography mass spectrometry; MRM, \\ multiple reaction monitoring; OA, oleic acid; PCa, prostate cancer; PUFA, \\ polyunsaturated fatty acid; SREBP-1c, sterol regulatory binding protein 1c;
}




\section{Clinical Relevance}

Fish oil, rich in n-3 polyunsaturated fatty acids (PUFAs), is a potential dietary supplement to lower prostate cancer risk. The mechanism of action of fish oil on prostate tumor growth inhibition remains largely unknown. While the n-3 fatty acids may exert important anticancer effects, the effects of other types of fatty acids such as oleic acid, a n-9 fatty acid and the main fatty acid in olive oil, are much less known. Roles of these PUFAs in prostate cancer have been investigated previously, however, conflicting results and the limitations in the experimental design make it clear that additional work needs to be conducted, especially at the cellular level, to better understand the pathways by which FO help reduce prostate cancer risk. 


\section{Abstract}

Purpose: The aim of this study is to investigate the role of $n-3$ and $n-9$ fatty acids in crucial processes involved in prostate cancer cell growth through a large-scale proteomic analysis.

Experimental design: We used a label-free protein quantification method to profile global protein expression of fish oil and oleic acid treated PCa cells and validated a panel of differentially expressed proteins by either Western blot or multiple reaction monitoring. Bioinformatic analysis was also performed to uncover the pathways involved in fatty acid metabolism.

Results: Fish oil, not oleic acid, suppresses prostate cancer cell viability. Assessment of fatty acid synthesis pathway activity also shows that oleic acid is a more potent inhibitor than fish oil on de novo fatty acid synthesis. Although fatty acid synthase activity decreases with fish oil treatment, the inhibition of its activity occurs over time while reduction in viability occurs within 24 hours. Bioinformatic analysis revealed the pathways altered by these fatty acid treatments.

Conclusions and clinical relevance: This study suggests that suppression of cell viability by fish oil is independent of fatty acid synthase and fish oil regulates prostate cancer cells through activation of other pathways depending upon length of exposure to fish oil. 


\section{Introduction}

Prostate cancer ( $\mathrm{PCa})$ is the second most common cancer in men [1]. The risk of $\mathrm{PCa}$ is currently one in six men $(16.7 \%)$ and the overall risk of death due to metastatic PCa is about $2.8 \%$ with the disease progressing slowly to advanced stage [2-4]. To date little is known about the etiological factors associated with PCa development and progression. There is increasing evidence suggesting that diet and lifestyle play a crucial role in PCa tumorigenesis $[5,6]$. However, a common consensus on which nutrients may be beneficial and which could be harmful is lacking, and this is further complicated by the contradictory reports about the role of different dietary components and nutrients on PCa prevention and progression [7-10].

Although two recent studies failed to support the role of fish oil (FO), which is rich in n-3 polyunsaturated fatty acids (PUFAs), especially docosahexaenoic acid (DHA, C22:6, $\mathrm{n}-3$ ) and eicosapentaenoic acid (EPA, C20:5, n-3), in protecting men against developing PCa $[10,11]$, it has long been viewed as a beneficial dietary supplement to lower the risk of PCa through suppressing PCa cell growth and inducing cell apoptosis, strengthening the effect of anti-tumor drug treatments to PCa cells [12-16]. These conflicting results and the limitations in the design of previous studies, make it clear that additional work needs to be conducted, especially at the cellular level, to better understand the pathways by which FO help reduce PCa risk.

Roles of other PUFAs in PCa have also been studied previously [17-20]. For example, an epidemiological study suggests that high ratio of $n-6 / n-3$ PUFAs uptake is positively associated with PCa risk [17]. Besides n-3 and n-6 FAs, other fatty acids (FAs) such as oleic acids (OA, C18:1, n-9), an n-9 monosaturated FA component, may also 
play important roles in reducing cancer risk. Unlike n-3 PUFAs, which are derived from limited sources (flaxseed, certain fish), n-9 fatty acids (such as OA) are one of the dominant components in most daily cooking oil. Results from studies investigating OA and cancer, however, are controversial. Some studies indicate OA has inhibitory effects on PCa cells [18], while others observed a higher OA/stearic ratio in untreated and more advanced PCa patients $[19,20]$. However, whether the high level of OA/stearic ratio is due to more endogenous OA production, dietary OA consumption, or the decrease level of stearic acid remains to be investigated. In the present study, we selected OA, a commonly consumed fatty acid, to be used in comparison with FO to identify the pathways activated or suppressed in PCa cells, specifically by FO and/or by OA. One of the downstream targets of dietary FAs is fatty acid synthase (FASN) [21], which is associated with development of multiple cancers and is currently being pursued as a therapeutic target [22]. Dietary PUFAs have been shown to suppress de novo synthesis of fatty acids via FASN through inhibition of the sterol regulatory binding protein 1c (SREBP-1c) $[23,24]$. Prostate tumor cells show markedly higher FASN expression compared to adjacent tissues $[25,26]$. FASN regulates a series of genes and pathways [27], some of which play critical roles in cell viability and its inhibitors have been reported to have anti-cancer effects [26, 28, 29]. N-3 PUFAs also modulate other molecules in PCa. One study suggests androgen receptor (AR) protein expression may be down-regulated by DHA treatment [30]. N-3 PUFAs also regulate enzymes involved in n-3 PUFA processing, for instance, cyclooxygenase 2 (COX2) [31].

To date the mechanism of FO's effects on PCa remains unknown. There is increasing evidence suggesting that FO may have multiple targets and thus its 
regulation may also be multi-level. This makes global proteome comparison an attractive approach. This study evaluated FO and OA's effects on PCa cell viability and FASN activity and sought to answer how the protein profiles are being regulated by FO or OA in a longitudinal manner with an LC-MS/MS method.

\section{Materials and methods}

\subsection{Cell line}

The PCa cell line, PC3 (ATCC), was grown in RPMI-160 medium (HyClone, Logan, UT, USA) supplemented with 10\% FBS (Sigma-Aldrich, St. Louis, MO, USA), $100 \mathrm{U} / \mathrm{ml}$ penicillin and $100 \mu \mathrm{g} / \mathrm{ml}$ streptomycin (Lonza, Walkersville, MD, USA) in $37^{\circ} \mathrm{C}$ with $5 \%$ $\mathrm{CO}_{2}$. For the androgen receptor positive PCa cell line, LAPC4, IMDM medium (HyClone, Logan, UT, USA) was used.

\subsection{FA treatment, chloroquine treatment and conditioned media collection}

FO and OA were purchased from KD Pharma Bexbach GmbH (Bexbach, Germany). FO capsules contained 90\% n-3 fatty acid ethyl ester (40\% EPA, 40\% DHA and 10\% other n-3 PUFAs). OA capsule contained $90 \%$ oleic acid ethyl ester. $100 \mathrm{mM}$ stock was made by dissolving fatty acids (FAs) in ethanol. When treating the cells, FAs-ethanol stock solution was mixed into complete medium at a 1:1000 ratio to a final concentration of $100 \mu \mathrm{M}$, while $0.1 \%$ ethanol was used in controls. For day- 6 treatment samples, the medium was changed four days after the treatment with a fresh medium containing FAs. In autophagy suppression assay, chloroquine diphosphate salt (Sigma-Aldrich, St. Louis, $\mathrm{MO}$, USA) was diluted to $8 \mu \mathrm{M}$ in medium. To harvest conditioned medium (CM), the growth medium was replace with serum-free medium, CM was harvested $24 \mathrm{hrs}$ later 
and cell debris was removed with centrifugation at 1,500 rpm. CM volume was normalized based on cell count with fresh serum-free medium to reach a same volume/cell number ratio among different treatments.

\subsection{Clonogenic cell survival assay}

Cells were seeded in 6-well plates (1,000 PC3 cells or 3,000 LAPC4 cells per well, respectively) and grown in complete medium with different FA treatments. Colonies formed at approximately 1 week for PC3 cells and 1.5 weeks for LAPC4 cells. Colonies were fixed and stained with crystal violet, washed and counted.

\subsection{Cell proliferation assay}

Cells were seeded in 96-well plates (1,000 PC3 cells or 6,700 LAPC4 cells per well, respectively) and grown in complete medium with different FA treatments. The MTT (3[4,5-dimethylthiazol-2-yl]-2,5 diphenyl tetrazolium bromide) assay was carried out by incubating cells with MTT (Sigma-Aldrich, St. Louis, MO, USA) for 4 hrs. DMSO was used to dissolve the formazan. Absorbance was measured at $570 \mathrm{~nm}$.

\subsection{Western blot}

Cell pellets were lysed by NP-40 lysis buffer supplemented with $1 \%$ protease inhibitor (Thermo-Fisher Scientific, Waltham, MA) and phosphatase inhibitor (SigmaAldrich, St. Louis, MO, USA). Laemmli buffer (Bio-Rad, Hercules, CA, USA) containing 5\% 2-mercaptoethonal (Fisher Scientific, Pittsburgh, PA, USA) was added to cell lysate supernatant and the mixtures were boiled to denature. Proteins were separated by SDS-PAGE gel then transferred to PVDF membrane (Bio-Rad, Hercules, CA, USA). Blocking was done in 5\% BSA (Roche, Indianapolis, IN, USA). PVDF membrane was incubated with primary antibodies anti-MSMP (Abnova, Walnut, CA, USA), anti- 
sequestosome-1 (Novus Biologicals, Littleton, CO, USA), anti-beta-actin (Fisher Scientific, Pittsburgh, PA, USA) and secondary antibodies (Santa Cruz Biotechnology, Santa Cruz, CA, USA) and Cell Signaling Technology (Danvers, MA, USA) sequentially. To detect LC3 I/II, cells were lysed by lysis buffer (1\% Triton-X100, $150 \mathrm{mM} \mathrm{NaCl,} 50$ $\mathrm{mM}$ Tris pH7.6), LC3 antibody were purchase from Thermo-Fisher Scientific (Waltham, MA, USA). Bands were detected by ECL ${ }^{\mathrm{TM}}$ Western Blotting Detection Reagents (Amersham $^{\mathrm{TM}}$, GE Healthcare, Piscataway, NJ, USA). Quantification was carried out using ImageJ, an open source image processing program.

\subsection{FA synthesis measurement}

Cells were incubated with complete culture medium with $1 \mu \mathrm{Ci} / \mathrm{ml} 1,2-{ }^{14} \mathrm{C}$-acetic acid sodium salt (PerkinElmer, Waltham, MA) was added to complete medium and cells were incubated for $2 \mathrm{hrs}$. The medium was discarded. For lipid extraction, $0.9 \mathrm{ml}$ chloroform/methanol $(2: 1, \mathrm{v} / \mathrm{v})$ mixture and $0.7 \mathrm{ml} 4 \mathrm{mM} \mathrm{MgCl} 2$ were added to the cells. The mixture was vortexed thoroughly and centrifuged at 10,000 rpm. The upper aqueous layer was removed and the lipid was maintained in bottom layer which is the chloroform fraction. Extraction was repeated for 3 times. Every sample was air dried in fume hood, then re-dissolved in $200 \mu \mathrm{l}$ chloroform and finally transferred to $10 \mathrm{ml}$ scintillation fluid Ultima Gold ${ }^{\mathrm{TM}} \mathrm{F}$ (PerkinElmer). Disintegration Per Minute (DPM) value was measured by TRI-CARB 2100TR liquid scintillation analyzer (Packard Instrument Company, Kennesaw, GA).

\subsection{Real-time PCR}

Cells were harvested and RNA extraction was carried out using RNeasy Plus Mini Kit (Qiagen, Valencia, CA, USA). Reverse transcription was performed using 
QuantiTect Reverse Transcription kit (Qiagen). Realtime PCR was performed on QuantStudio 12K Flex (Applied Biosystems, Foster City, CA, USA) and the protocol was designed according to Taqman Gene Expression Assay (Applied Biosystems, Foster City, CA, USA). Sequestosome-1 (SQSTM1) was labeled with reporter dye FAM.

Glucuronidase $\beta$ (GUSB), which serves as internal standard was labeled with reporter

dye VIC. Realtime PCR primers and probes targeting SQSTM1 (assay ID

Hs01061917_g1), GUSB (assay ID: Hs00939627_m1), as well as Mastermix (the reaction solution), were designed and purchased from Applied Biosystems.

\subsection{Mass spectrometry}

For global unbiased proteomic analysis, cell pellets were lysed in $8 \mathrm{M}$ urea and 10 $\mathrm{mM}$ dithiothreitol (DTT) and then sonicated on ice. Equal amount of chicken lysozyme was spiked into each sample at a final concentration of $0.5 \%(\mathrm{w} / \mathrm{w})$, serving as a quality assurance (QA)/quality control (QC) reference. Reduction/alkylation of cysteine residues was done by incubating the cell extracts with acetonitrile (ACN)/iodoethanol/triethylphosphine (195:4:1, v/v/v) mixture. Samples were dried in a speed vacuum concentrator (Genevac, NY, USA) and resuspended in $310 \mu \mathrm{l}$ of $100 \mathrm{mM}$ ammonium bicarbonate, followed by overnight trypsin (Worthington, Lakewood, NJ) digestion (trypsin:protein=1:25). Desalting was carried out by repeating $\mathrm{ACN}-\mathrm{H}_{2} \mathrm{O}$ with $0.1 \%$ formic acid wash in Silica C18 MacroSpin Column (The Nest Group, Southborough, MA, USA). The resulting peptides were eluted by $80 \% \mathrm{ACN}$ in last step and speed-vac dried. Samples were resuspended in $5 \% \mathrm{ACN}$ in water with $0.1 \%$ formic acid and filtered through Ultrafree-MC Centrifugal filters (EMD Millipore, Billerica, MA). Peptides were eluted with a linear gradient from $5 \%$ to $80 \%$ ACN developed over 120 
min at a flow rate of $50 \mu \mathrm{l} / \mathrm{min}$, and effluent was electro-sprayed into the LTQ Orbitrap Velos Pro mass spectrometer (Thermo-Fisher Scientific, Waltham, MA, USA). Two injections of each sample were carried out randomly. X!tandem algorithms were applied to search the acquired data against UniProt human database (released in April 2013). Peptides and proteins were validated using PeptideProphet and ProteinProphet in the Trans-Proteomics Pipeline (TPP, v.4.6). All the proteins listed in this study have protein probability $>99.8 \%$ and peptide probability $>80 \%$. Label-free quantification was carried out using IdentiQuantXL ${ }^{\mathrm{TM}}$ [32].

\subsection{MRM-based validation}

Sample preparation, including cell lysis, reduction/alkylation and digestion was carried out the same way as that in proteomic discovery. Mass spectrometric analyses were performed on an AB/SCIEX 4000 Qtrap mass spectrometer (Sciex, Framingham, MA, USA) interfaced with a Dionex U-3000 UHPLC system (Thermo-Fisher Scientific, Waltham, MA, USA). Peptides were injected on an ODS-100V C18 column (1.0mm x 150mm, Tosoh Biosciences, South San Francisco, CA, USA) and eluted with a linear gradient from 5 to $80 \%$ acetonitrile over $32 \mathrm{~min}$ at a flow rate of $50 \mu \mathrm{l} / \mathrm{min}$. The parameters were as follows: curtain gas of $40.0 \mathrm{psi}$, collision gas of $7 \mathrm{psi}$, ion spray voltage of $5500 \mathrm{~V}$, temperature set at $500{ }^{\circ} \mathrm{C}$, ion source gas 1 of $50.0 \mathrm{psi}$, ion source gas 2 of 40.0 psi. Entrance potential is set on $10.0 \mathrm{~V}$; collision cell exit potential is 12.0 V. Data acquisition was carried out using the AB/SCIEX Analyst ${ }^{\circledR}$ software. For MRM development, the Skyline software was first used to generate candidate peptides and theoretical transitions for each peptide. PC3 cell lysate was tested to select the best experimental peptide and transitions, which could be stably detected and also had high 
peak intensity. One specific transition was monitored for each target peptide, which represents one specific protein. Each monitored transition was confirmed by spiking in varied concentration of the corresponding synthetic peptides. For FSCN1, a transition for target peptide ${ }^{23}$ YLTAEAFGFK $^{32}$ was monitored: $\mathrm{m} / \mathrm{z} 573.8\left(\mathrm{M}+2 \mathrm{H}^{+}\right) \rightarrow \mathrm{m} / \mathrm{z} 870.4$. For CANX, a transition for target peptide ${ }^{78}$ GTLSGWILSK ${ }^{87}$ was monitored: $\mathrm{m} / \mathrm{z} 531.3$ $\left(\mathrm{M}+2 \mathrm{H}^{+}\right) \rightarrow \mathrm{m} / \mathrm{z}$ 790.4. For MSMP, a transition for target peptide ${ }^{52} \mathrm{YFTLGESWLR}{ }^{61}$ was monitored: $\mathrm{m} / \mathrm{z} 636.3\left(\mathrm{M}+2 \mathrm{H}^{+}\right) \rightarrow \mathrm{m} / \mathrm{z}$ 747.4. For SCP2, a transition for target peptide ${ }^{48}$ IGGIFAFK ${ }^{55}$ was monitored: $\mathrm{m} / \mathrm{z} 426.8\left(\mathrm{M}+2 \mathrm{H}^{+}\right) \rightarrow \mathrm{m} / \mathrm{z}$ 739.4. A transition for chicken lysozyme, which serves as external standard, target peptide ${ }^{40}$ GYSLGNWVCAAK ${ }^{61}$ was monitored: $\mathrm{m} / \mathrm{z} 656.8\left(\mathrm{M}+2 \mathrm{H}^{+}\right) \rightarrow \mathrm{m} / \mathrm{z}$ 892.4. We also monitored a transition for RPL6, which serves as the internal standard, target peptide: ${ }^{211} \mathrm{HLTDAYFK}^{218}, \mathrm{~m} / \mathrm{z} 497.8$ $\left(\mathrm{M}+2 \mathrm{H}^{+}\right) \rightarrow \mathrm{m} / \mathrm{z}$ 744.4. Declustering potential (DP) and collision energy (CE) were optimized for each peptide. Scan time for each peptide was $120 \mathrm{msec}$ and total scan time was $0.875 \mathrm{sec}$. Quantification of target peptide was performed using Skyline software to calculate area under curve (AUC) of extracted ion chromatograms.

\section{RESULTS}

The aim of this study was to better understand the pathways involved in FO inhibition of PCa cell viability and to further investigate FO and OA's effects in global protein expression. We compared global protein expression profiles of PC3 cells treated with $\mathrm{FO}$ and $\mathrm{OA}$ at two different time points. Protein expression data presented here clearly demonstrate that FA-mediated molecular changes are not directly linked to FASN activity and the function of FAs may likely be mediated through multiple targets. 


\subsection{FO, not OA inhibits PCa cell growth}

A MTT cell proliferation assay was performed to assess cell viability upon PUFA treatment. Shown in Fig. 1A, FO, not OA, suppresses cell viability. A significant difference in cell growth $(\mathrm{FO}=74.8 \% \mathrm{C}, \mathrm{p}=0.0105)$ was observed after 24 hours in the FO treated cells but not in $O A$ group $(O A=101.4 \% C, p=0.5493)$. This observation was confirmed by clonogenic assays, in which colony density was significantly lower with FO treatment (Fig. 1B). Clonogenic cell survival assays were also performed on androgen receptor (AR) positive LAPC4 cells (data not shown). FO treatment demonstrated significant inhibition of colony formation $(p<0.05)$ with no LAPC4 colonies observed in FO treated group after 1.5 weeks. Again, the OA group showed no significant change as compared to control group $(p=0.2842)$. During colony formation, vacuoles were observed in the FO treated cells, which remained single cells and stopped proliferating (Fig. 1C).

\subsection{Both FO and OA inhibit FA synthesis and OA is a more potent inhibitor than} FO

De novo FA synthesis was measured in the FO and OA treated groups after three days and six days treatment. At day three there was no change in fatty acid synthesis with fish oil treatment (Fig. 2A), while a $30.97 \%(p=0.0001)$ suppression was observed in the OA group. After six days of treatment, a significant FA synthesis suppression was observed in both FO $(27.11 \%, p<0.05)$ and OA $(49.21 \%, p<0.0001)$ groups (Fig. 2B). Additionally, OA's FA synthesis level was lower than FO (OA=69.7\%FO, $p=0.0301)$, which suggests that OA is a more potent FA synthesis inhibitor than FO. This 
observation also implicates that the viability inhibition effect of FO on PC3 cells may not be directly linked to FA synthesis.

\subsection{The differential proteome profile in prostate cancer cells treated with FO or}

OA

LC-MS-based label-free protein quantification was performed to investigate the longitudinal regulation of FO and OA on PCa cells. PC3 cells were plated at $\sim 170,000$ cells/plate on $100-\mathrm{mm}$ plates with the complete medium with $0.1 \%$ ethanol, $100 \mu \mathrm{M} \mathrm{FO}$, or $100 \mu \mathrm{M}$ OA, respectively. Cells were harvested on day 1 and day 6 after treatment for MS analysis. A total of 1,478 proteins were identified with protein probability $>99.8 \%$ and peptide probability $>80 \%$. Protein and peptide probabilities were produced through calculation by TPP. Random sequences were set up serving as decoy sequences. A protein identification probability $>99.8 \%$ corresponds to a FDR of $<1 \%$, which is a commonly used cutoff in the proteomics field.

Protein expression comparison was conducted based on treatment conditions (control, FO, and OA) and time-course (day-1 and day-6). Three hundred and twelve proteins were positively identified based on p-values less than 0.05 . As indicated in the Supplementary Table S1, majority of the technical variations are under $10 \%$ with a few outliers based on the internal control (chicken lysozyme) data and the overall \% CV is below $13 \%$ (Supplementary Table S1). This is what we expected from this type of global protein profiling experiment with the platform we applied. The data was further filtered by FDR-adjusted $p$ value ( $q$ value cut off $=0.05$ ) and the expression levels of 127 proteins were significantly changed upon treatment (Supplementary Table S2). Principal component analysis (PCA) was performed on this 127-protein dataset that consisted of 
6 treatment groups with 5 replicates in each group. Prolonged FO treated group (FO-6 day) was a distinct group indicated by the red circle, separated from the other treatments (Fig.3A). The blue and yellow circles indicate there were fewer protein changes among treatments in day-1 and between control and OA group in day-6. By comparing each $\mathrm{FO}$ or $\mathrm{OA}$ group to its same-day corresponding control group, 73 proteins showed significant changes $(p \leq 0.05)$. $\log _{2}($ Ratio $<F O / C>)$ and $\log _{2}($ Ratio $<\mathrm{OA} / \mathrm{C}>)$ values of protein entries were clustered by one minus Pearson Correlation (Gene E online analysis platform, http://www.broadinstitute.org/cancer/software/GENE-E/). Three major clusters were generated (shown in Fig.3B). Cluster A contained 7 proteins (encoded by 6 genes) (Table 1), including SCP2 and acyl-coenzyme A oxidase which participate in fatty acid transport and metabolism [33,34]; coactosin-like protein is reported to be involved in PUFA metabolism [35]. Cluster B showed that five of the six proteins (encoded by 4 genes) were significantly elevated in the FO group one day after treatment, and protein expression levels were higher than the OA group. Among these proteins, sequestosome-1 (SQSTM1) and heat shock $70 \mathrm{kDa}$ protein 1A/1B (HSPA1A) were identified and have roles in the autophagy process $[36,37]$. In cluster $\mathrm{C}$, which consisted of 60 protein entries, no significant changes were observed in $\mathrm{FO}$ and $\mathrm{OA}$ groups in the first day of treatment. However, on day-6, FO-treated and OA-treated groups showed divergent protein expression changes. In the FO-treated group, 53 out of 60 proteins showed a significant decrease in expression while in the OA-treated group, the majority of differentially regulated proteins were elevated and eight of the changes were significant. Ingenuity ${ }^{\circledR}$ pathway analysis (IPA) indicated that cell 
movement, growth and proliferation, cell development, cell death/survival, and carbohydrate metabolism were the most significant molecular and cellular functions involved (Table 2), while glycolysis and gluconeogenesis were the top canonical pathways. Four enzymes from cluster C: phosphoglycerate kinase 1 (PGK1), glyceraldehyde-3-phosphate dehydrogenase (GAPDH), pyruvate kinase isozymes M1/M2 (PKM) and its isoform M1 (PK) and glucose-6-phosphate isomerase (GPI) were all significantly decreased in expression in FO-6-day treated group, but have little change in the OA-6-day treated group (Fig. 4).

In combination with IPA, the proteins with significant expression changes were selected based on the following criteria: 1) protein probability $>99.8 \%$; 2 ) two or more unique peptide sequences identified with high confidence; 3) within every group, ANOVA $p$-value $\leq 0.05$, q-value $\leq 0.05 ; 4$ ) multiple comparison test $p$-value $\leq 5 / \mathrm{N}_{\text {total }}$ tests-; 5) protein frequency (occurrence in all injections) $>50 \% ; 6$ ) association with cancer,

prostate, or lipid processing; 7) absolute fold change $>1.3$. Under these stringent criteria, we identified only one differentially regulated protein, sequestosome-1 (SQSTM-1), in the day-1 treatment group. SQSTM-1 was elevated (1.7-1.8 fold) in the FO group compared to control and OA group. In the 6-day treatment comparison, the expression levels of 4 proteins were significantly altered as compared to the controls (Table 3 ).

\subsection{Key differentially expressed protein validation}

Both Western blot and MRM-based methods were used for validation studies to confirm the differential protein expression observed in the global discovery study. Since the majority of these proteins were in the day- 6 treatment group, we used an independent set of day- 6 samples for validation studies. In MRM, for each selected 
protein, one unique peptide and one pair of precursor and product ions (MRM transitions) were selected. Relative quantity and specificity of these MRM peptides/transitions are illustrated in Supplementary Figures S1, S2, and S3. Synthetic standard peptides were first tested to confirm the transition ion pairs derived from Skyline. Results showed that among the 4 proteins changed in the day- 6 treatment group, prostate-associated microseminoprotein (MSMP) was found significantly decreased when the FO treatment group was compared to control $(63.3 \%$ lower expression in the FO group, $p=0.0242$ ). Down-regulation of MSMP in the OA treated group was not significant (28.4\% lower expression, $p=0.3474)$, (Fig. 5A). Since MSMP is also a secreted protein, we tested expression in conditioned medium (CM) of PC3 cell grown in the presence of FO, OA or vehicle. Western blot results show that secreted MSMP in CM appears as a single band around 16kD. FO treated PC3 cells secreted less MSMP compared to control $(p=0.0096)$ or OA group $(p=0.029)$ (Fig. 6F $\&$ 6G).

The other protein, sterol carrier protein 2 (SCP2), was also found to be significantly up-regulated in the FO treated group compared to control (47.1\% over-expressed in FO group, $p=0.001)$, or OA (27.8\%FO, $p=0.0113)$ (Fig. 5B). However, unlike the global proteome results, there was no significant change between $\mathrm{OA}$ and $\mathrm{C}$. No significant changes were observed in spiked-in chicken lysozyme and RPL6, which served as QA/QC samples (constant amount among three groups observed) (Fig. 5C \& 5D).

Sequestosome 1 (SQSTM1), a protein involved in autophagy, was found to be overexpressed in FO group one day after the treatment in our global proteomic study. Western blot results were consistent with the LC/MS results that demonstrated SQSTM1 levels in the FO group were 2.1-fold higher than that in the control group 
$(p=0.042)($ Fig. 6A \& 6B). Although the proteomic analysis also showed a higher level of expression in FO group when FO and OA groups were compared (fold-change=2.35), the change, however, was not statistically significant $(p=0.0867)$ based on our $p=0.05$ cutoff. There was no significant difference between OA and control. Real-time PCR results showed that at the transcriptional level, mRNA expression of SQSTM1 in FO treated group was higher than both control (fold-change $=2.16, p=0.003$ ) and OA treated group (fold-change=2.26, $p=0.003$ ) (Fig. 6C). To further investigate the function of autophagy, autophagy inhibitor chloroquine (CQ) was applied to cells with different FA treatment, and LC3 I/II levels were measured by Western blot. The results indicated that, under the condition of autophagy suppression induced by CQ, LC3 II levels accumulated significantly (two-way ANOVA, $p=0.0005$ ) while LC3 I levels were not affected. However, no significant LC3 II changes were observed among FA treatment groups (two-way ANOVA, p=0.7853) (Fig. 6D, 6E), suggesting that autophagy may not be significantly inhibited under this experimental condition.

\section{DISCUSSION}

Saturated fatty acids, OA, and n-6 PUFA are main components of daily cooking oil, including corn, peanut or sunflower seed oil. Except for flaxseed oil (alpha-linolenic acid, ALA) and some fish (DHA and EPA), n-3 PUFA, one of the essential FAs, exist as a very small portion of dietary oil intake. Proteomic studies of this sort have been carried out to investigate the global protein level change under different dietary FA consumption. However, most of these studies used two-dimensional gel electrophoresis approaches with the study objectives varying from insulin resistance to colon inflammation [38-44]. 
Large-scale proteomic studies on dietary fish oil effect on PCa using the label-free protein quantification method have not yet been reported.

In this study, we investigated the different molecular pathways of OA and FO action in PCa cells. Previous investigations of n-3 PUFAs and PCa have pointed to some of the potential mechanisms of FO on PCa growth inhibition. One study detected downregulated AR protein expression in LNCaP cells after 10 weeks continuous $20 \mu \mathrm{M}$ DHA treatment [30], while another study did not detect a AR protein change in LNCaP cells treated by DHA at concentrations as high as $180 \mu \mathrm{M}$ for 24 to $36 \mathrm{hrs}$ [14], suggesting differential regulatory changes over time may have occurred during the treatment periods. One point worth noting is that the concentration of the fatty acids used for this study is based on our preliminary experiments and other reported studies $[13,45,46]$. At $100 \mu \mathrm{M}, \mathrm{FASN}$ activity is significantly reduced by OA while cell viability remains unaffected. However, in vivo human exposure to this concentration of FAs may never reach $100 \mu \mathrm{M}$ due to its poor water solubility and lipid intake limitation. Thus the actual exposure of the cells to FAs may be much lower than the calculated $100 \mu \mathrm{M}$ concentration.

Our results suggest that only $\mathrm{FO}$, not $\mathrm{OA}$, suppresses $\mathrm{PC} 3$ cell viability, and this inhibition occurred as early as one day after the treatment. Hierarchy analysis of global proteomic data generated a group of proteins in which the majority of proteins were increased after 24-hr FO treatment. Specifically, both sequestosome-1 (SQSTM1), heat shock $70 \mathrm{kDa}$ proteins $\mathrm{A} 1 \mathrm{~A} / \mathrm{A} 1 \mathrm{~B}$ are connected to autophagy $[36,37]$ and both were significantly elevated one day after FO treatment but with no change in the OA treated group. This result indicates FO, not OA, triggers rapid stress-responding reactions, 
which may explain the early event of viability inhibition. The elevated expression of SQSTM1, an autophagy associated protein, was also validated by Western Blot (Fig. 6A \& 6B). Tumor cells activate autophagy in response to cellular stress and/or increased metabolic demands related to rapid cell proliferation. In the dynamic process of autophagy, SQSTM1 is required for the degradation of polyubiquitinated substrates, and SQSTM1 itself is also degraded during this process. The accumulating SQSTM1 level implicates a possible inhibition of autophagy, which in turn causes the accumulation of SQSTM1 that should be otherwise degraded in a normal autophagy process, suppressing the growth of the tumor cells. Consistent with our result, one study also found elevated SQSTM1 12-hr after the treatment of EPA in promyelocytic cells [47]. Conversely, Shin et al. showed that autophagy, in this case acting as a tumor suppressor, was activated by DHA and played a critical role in apoptosis of PC3 cells induced by DHA [48]. However, in investigating the cause of SQSTM1 elevation under FO treatment, we found this increased protein expression was most likely regulated by transcriptional mechanism rather than reduced post-translational degradation, implicating other transcription regulator(s) may be involved. It has been previously reported that oxidation stress can induce SQSTM1 at the transcriptional level, and SQSTM1 triggers antioxidant effects [49]. Since FO was found to induce oxidation stress and cytotoxicity [50], we suggest that the observed increase of SQSTM1 might take place at the stage when cells are starting to respond to oxidation stress.

Our study suggests that although OA suppressed FA synthesis rate to near $50 \%$ on day 6, FASN protein level showed no significant change in the OA group compared to control. One study in C6 glioma cells suggested OA was a stronger inhibitor than n-3 
PUFA and other FAs in FA synthesis [51]. Consistent with their result and another study in 1999 [24], we found no protein level changes in FASN expression. Interestingly, they discovered that OA down-regulated the expression of another key enzyme in FA synthesis, acetyl-coenzyme A carboxylase, which is involved in the formation of malonyl-CoA. In contrast, FASN expression levels in FO treated group are decreased compared to OA group (t-test, $p=0.05$, multiple comparison test $p=0.1$ ), indicating additional transcriptional level modulation of FASN was triggered by FO.

Our quantitative global proteomic study also revealed that a group of glycolysis/gluconeogenesis enzymes were changed in FO treated cells and four of the enzymes were significantly down-regulated in the 6-day treatment group. Although most of the decreased enzymes are shared by both glycolysis and gluconeogenesis, PKM specifically catalyzes a rate-limiting step in glycolysis. In PCa, glucose is not a major bioenergy source as in many other cancer types [52], therefore rendering FDG-PET imaging ineffective for PCa tumor imaging [53]. However, a study based on NIH dbEST database showed PCa tissue has 9 out of 10 glycolysis genes overexpressed compared to normal tissue [54]. Additionally, some glycolytic enzymes, PKM and PGAM1, are expressed differently between PCa and normal tissue by isoform or post-translational modification (PTM) level [55]. PCa cell sensitivity to stress is enhanced when glycolysis is suppressed [56]. This suggests glycolysis still plays a role in PCa even though the glucose uptake is low. Expression of hepatic pyruvate kinase [57] and glucose-6phosphate dehydrogenase [58] were also reportedly blocked by n-6 or n-3 PUFAs. One of the possible mechanisms is through prevention of chREBP translocating to nuclei [59]. In the context of cancer cells, the inhibition may be mediated by liver kinase B1 
(LKB) [60]. Effects of PUFAs on glycolysis are tissue specific so that in skeletal muscle cells, the process is reversed [45]. Our study indicates FO may suppress glycolysis by decreased multiple enzyme expression, suggesting that in the PC3 cell line, de novo FA synthesis decreases in later time points may be due to the lower pyruvate supplied by glycolysis. To the contrary, prolonged OA treatment does not suppress glycolytic enzyme expression and we therefore conclude that FO and OA may inhibit FA synthesis through different pathways, which are dependent or independent of glycolysis down-regulation, respectively.

Besides involvement of glycolytic enzymes, additional potential targets of FO were investigated and validated in independent sets of samples. Among the five candidate proteins in the 6-day treatment arm, the increase of SCP2 and decrease of MSMP was validated by MRM. In the global study, SCP2 was elevated in both FO and OA, with the FO group demonstrating significantly higher than OA and control. This result was confirmed by Western Blot analysis, although the SCP2 increase in OA group was not significant. Functionally it is expected that this lipid carrier protein expression would increase when exogenous FAs are added to the medium. The other protein, consistent in validation experiments, MSMP, is a highly conserved protein and belongs to betamicroseminoprotein family and its expression was observed in both benign and tumor tissues although immunohistochemical analysis demonstrated that tumor MSMP expression is different from the pattern in benign tissue [61]. As a recently identified protein, MSMP has not been well studied. One report suggested MSMP's role in inflammation as a ligand binding to $\mathrm{CC}$ chemokine receptor $2 \mathrm{~B}(\mathrm{CCR} 2 \mathrm{~B})$ and acts as a chemoattractant, which induces the migration of peripheral blood monocytes (PBM) and 
peripheral blood lymphocyte (PBL) cells but not polymorphonuclear neutrophils (PMN) cells [61], indicating a pro-inflammation role of MSMP. MSMP was also found to stimulate $p$-ERK, which plays a central signaling role in cancer progression $[62,63]$. Our results demonstrate that FO can lower MSMP levels, offering an additional explanation for the anti-inflammation action of n-3 FAs in prostate cancer. Taken together, FO and OA both suppress FA synthesis activity but only FO inhibits cell survival. Global proteomic studies and following validation demonstrated that FO modulates protein levels of SQSTM1, MSMP, and SCP2 in PCa cells, suggesting a possible longitudinal regulation by FO: inducing cytotoxicity after short treatment; suppressing inflammation pathways and inhibiting the glycolytic pathway in prolonged treatment.

The authors have declared no conflict of interest. 


\section{References}

1. King, A. J., Evans, M., Moore, T. H., Paterson, C., et al., Prostate cancer and supportive care: a systematic review and qualitative synthesis of men's experiences and unmet needs. Eur. J. Cancer Care 2015, Jan 29. Epub ahead of print.

2. Jemal, A., Thomas, A., Murray, T., and Thun, M., Cancer statistics, 2002. CA Cancer J. Clin. 2002, 52, 23-47.

3. Salinas, C. A., Tsodikov, A., Ishak-Howard, M., and Cooney, K. A., Prostate cancer in young men: an important clinical entity. Nat. Rev. Urol. 2014, 11, 317-323.

4. Crawford, E. D., Prostate Cancer Awareness Week: September 22 to 28, 1997. CA Cancer J. Clin. 1997, 47, 288-296.

5. Hu, J., La Vecchia, C., Gibbons, L., Negri, E., et al., Nutrients and risk of prostate cancer. Nutr. Cancer 2010, 62, 710-718.

6. Aronson, W. J., Barnard, R. J., Freedland, S. J., Henning, S., et al., Growth inhibitory effect of low fat diet on prostate cancer cells: results of a prospective, randomized dietary intervention trial in men with prostate cancer. J. Urol. 2010, 183, 345-350.

7. Szymanski, K. M., Wheeler, D. C., and Mucci, L. A., (2010) Fish consumption and prostate cancer risk: a review and meta-analysis. Am. J. Clin. Nutr. 2010, 92, 12231233.

8. Vissapragada, S., Ghosh, A., Ringer, L., Salinas, P., et al., Dietary n-3 polyunsaturated fatty acids fail to reduce prostate tumorigenesis in the PB-ErbB-2 $x$ Pten(+/-) preclinical mouse model. Cell Cycle 2010, 9, 1824-1829.

9. Berquin, I. M., Min, Y., Wu, R., Wu, J., et al., Modulation of prostate cancer genetic risk by omega-3 and omega-6 fatty acids. J. Clin. Invest. 2007, 117, 1866-1875. 
10. Brasky, T. M., Darke, A. K., Song, X., Tangen, C. M., et al., Plasma phospholipid fatty acids and prostate cancer risk in the SELECT trial. J. Natl. Cancer Inst. 2013, $105,1132-1141$.

11. Lovegrove, C., Ahmed, K., Challacombe, B., Khan, M. S., et al., Systematic review of prostate cancer risk and association with consumption of fish and fish-oils: analysis of 495,321 participants. Int. J. Clin. Pract. 2015, 69, 87-105.

12. Hu, Y., Sun, H., Owens, R. T., Gu, Z., et al., Syndecan-1-dependent suppression of PDK1/Akt/bad signaling by docosahexaenoic acid induces apoptosis in prostate cancer. Neoplasia 2010, 12, 826-836.

13. Shaikh, I. A., Brown, I., Schofield, A. C., Wahle, K. W., et al., Docosahexaenoic acid enhances the efficacy of docetaxel in prostate cancer cells by modulation of apoptosis: the role of genes associated with the NF-kappaB pathway. The Prostate $2008,68,1635-1646$.

14. Chung, B. H., Mitchell, S. H., Zhang, J. S., and Young, C. Y., Effects of docosahexaenoic acid and eicosapentaenoic acid on androgen-mediated cell growth and gene expression in LNCaP prostate cancer cells. Carcinogenesis 2001, 22, 1201-1206.

15. Hsing, A. W., Essential fatty acids and prostate cancer: an emerging hypothesis? Cancer Epidemiol. Biomarkers Prev. 1996, 5, 859-860.

16. Karmali, R. A., Eicosanoids in neoplasia. Prev. Med. 1987, 16, 493-502.

17. Williams, C. D., Whitley, B. M., Hoyo, C., Grant, D. J., et al., A high ratio of dietary n6/n-3 polyunsaturated fatty acids is associated with increased risk of prostate cancer. Nutr. Res. 2011, 31, 1-8. 
18. Hughes-Fulford, M., Chen, Y., and Tjandrawinata, R. R., Fatty acid regulates gene expression and growth of human prostate cancer PC-3 cells. Carcinogenesis 2001, 22, 701-707.

19. Persad, R. A., Gillatt, D. A., Heinemann, D., Habib, N. A., et al., Erythrocyte stearic to oleic acid ratio in prostatic carcinoma. Brit. J. Urol. 1990, 65, 268-270.

20. Kositsawat, J., Flanigan, R. C., Meydani, M., Choi, Y. K., et al., The ratio of oleic-tostearic acid in the prostate predicts biochemical failure after radical prostatectomy for localized prostate cancer. J. Urol. 2007, 178, 2391-2396.

21. Rudolph, M. C., Monks, J., Burns, V., Phistry, M., et al., Sterol regulatory element binding protein and dietary lipid regulation of fatty acid synthesis in the mammary epithelium. Am. J. Physiol. Endocrinol. Metabol. 2010, 299, E918-927.

22. Mashima, T., Seimiya, H., and Tsuruo, T., De novo fatty-acid synthesis and related pathways as molecular targets for cancer therapy. Brit. J. Cancer 2009, 100, 13691372.

23. Teran-Garcia, M., Adamson, A. W., Yu, G., Rufo, C., et al., Polyunsaturated fatty acid suppression of fatty acid synthase (FASN): evidence for dietary modulation of NF-Y binding to the Fasn promoter by SREBP-1c. Biochem. J. 2007, 402, 591-600.

24.Xu, J., Nakamura, M. T., Cho, H. P., and Clarke, S. D., Sterol regulatory element binding protein-1 expression is suppressed by dietary polyunsaturated fatty acids. A mechanism for the coordinate suppression of lipogenic genes by polyunsaturated fats. J. Biol. Chem. 1999, 274, 23577-23583. 
25. Rossi, S., Graner, E., Febbo, P., Weinstein, L., et al., Fatty acid synthase expression defines distinct molecular signatures in prostate cancer. Mol Cancer Res. 2003,1, 707-715.

26. Pizer, E. S., Pflug, B. R., Bova, G. S., Han, W. F., et al., Increased fatty acid synthase as a therapeutic target in androgen-independent prostate cancer progression. The Prostate 2001, 47, 102-110.

27. Coleman, D. T., Bigelow, R., and Cardelli, J. A., Inhibition of fatty acid synthase by luteolin post-transcriptionally down-regulates c-Met expression independent of proteosomal/lysosomal degradation. Mol. Cancer Ther. 2009, 8, 214-224.

28. Grube, S., Dunisch, P., Freitag, D., Klausnitzer, M., et al., Overexpression of fatty acid synthase in human gliomas correlates with the WHO tumor grade and inhibition with Orlistat reduces cell viability and triggers apoptosis. J. Neuro. Oncol. 2014, 118, 277-287.

29. Chen, H. W., Chang, Y. F., Chuang, H. Y., Tai, W. T., et al., Targeted therapy with fatty acid synthase inhibitors in a human prostate carcinoma LNCaP/tk-luc-bearing animal model. Pros. Cancer Pros. Dis. 2012, 15, 260-264.

30. Friedrichs, W., Ruparel, S. B., Marciniak, R. A., and deGraffenried, L., Omega-3 fatty acid inhibition of prostate cancer progression to hormone independence is associated with suppression of mTOR signaling and androgen receptor expression. Nutr. Cancer 2011, 63, 771-777.

31. Kobayashi, N., Barnard, R. J., Henning, S. M., Elashoff, D., et al., Effect of altering dietary omega-6/omega-3 fatty acid ratios on prostate cancer membrane 
composition, cyclooxygenase-2, and prostaglandin E2. Clin. Cancer Res. 2006, 12, $4662-4670$.

32.Lai, X., Wang, L., Tang, H., and Witzmann, F. A., A novel alignment method and multiple filters for exclusion of unqualified peptides to enhance label-free quantification using peptide intensity in LC-MS/MS. J. Proteome Res. 2011, 10, $4799-4812$.

33. Filipp, F. V., and Sattler, M., Conformational plasticity of the lipid transfer protein SCP2. Biochemistry 2007, 46, 7980-7991.

34. Kim, S., Sohn, I., Ahn, J. I., Lee, K. H., et al., Hepatic gene expression profiles in a long-term high-fat diet-induced obesity mouse model. Gene 2004, 340, 99-109.

35. Rakonjac, M., Fischer, L., Provost, P., Werz, O., et al., Coactosin-like protein supports 5-lipoxygenase enzyme activity and up-regulates leukotriene A4 production. Proc. Natl. Aca. Sci. USA. 2006, 103, 13150-13155.

36. Pursiheimo, J. P., Rantanen, K., Heikkinen, P. T., Johansen, T., et al., Hypoxiaactivated autophagy accelerates degradation of SQSTM1/p62. Oncogene 2009, 28, $334-344$.

37. Minoia, M., Boncoraglio, A., Vinet, J., Morelli, F. F., et al., BAG3 induces the sequestration of proteasomal clients into cytoplasmic puncta: implications for a proteasome-to-autophagy switch. Autophagy 2014, 10, 1603-1621.

38. Rangel-Zuniga, O. A., Camargo, A., Marin, C., Pena-Orihuela, P., et al., Proteome from patients with metabolic syndrome is regulated by quantity and quality of dietary lipids. BMC Genomics 2015, 16, 509. 
39. Huang, C. Y., Chen, W. M., Tsay, Y. G., Hsieh, S. C., et al., Differential regulation of protein expression in response to polyunsaturated fatty acids in the liver of apoEknockout mice and in HepG2 cells. J. Biomed. Sci. 2015, 22, 12.

40. Jimenez-Gomez, Y., Cruz-Teno, C., Rangel-Zuniga, O. A., Peinado, J. R., et al., Effect of dietary fat modification on subcutaneous white adipose tissue insulin sensitivity in patients with metabolic syndrome. Mol. Nutr. Food Res. 2014, 58, 2177-2188.

41.Park, J., Yamaura, T., Kawamoto, J., Kurihara, T., et al., Reciprocal modulation of surface expression of annexin A2 in a human umbilical vein endothelial cell-derived cell line by eicosapentaenoic acid and docosahexaenoic acid. PLOS One 2014, 9, e85045.

42. Ahmed, A. A., Balogun, K. A., Bykova, N. V., and Cheema, S. K., Novel regulatory roles of omega-3 fatty acids in metabolic pathways: a proteomics approach. Nutr. Metabol. 2014, 11, 6.

43. Camargo, A., Rangel-Zuniga, O. A., Pena-Orihuela, P., Marin, C., et al., Postprandial changes in the proteome are modulated by dietary fat in patients with metabolic syndrome. J. Nutr. Biochem. 2013, 24, 318-324.

44. Cooney, J. M., Barnett, M. P., Brewster, D., Knoch, B., et al., Proteomic analysis of colon tissue from interleukin-10 gene-deficient mice fed polyunsaturated Fatty acids with comparison to transcriptomic analysis. J. Proteome Res. 2012, 11, 1065-1077.

45. Vaughan, R. A., Garcia-Smith, R., Bisoffi, M., Conn, C. A., et al., Conjugated linoleic acid or omega 3 fatty acids increase mitochondrial biosynthesis and metabolism in skeletal muscle cells. Lipids Health Dis. 2012, 11, 142. 
46. Chiang, K. C., Persons, K. S., Istfan, N. W., Holick, M. F., et al., Fish oil enhances the antiproliferative effect of 1alpha,25-dihydroxyvitamin D3 on liver cancer cells. Anticancer Res. 2009, 29, 3591-3596.

47. Slagsvold, J. E., Pettersen, C. H., Follestad, T., Krokan, H. E., et al., The antiproliferative effect of EPA in HL60 cells is mediated by alterations in calcium homeostasis. Lipids 2009, 44, 103-113.

48. Shin, S., Jing, K., Jeong, S., Kim, N., et al., The Omega-3 Polyunsaturated Fatty Acid DHA Induces Simultaneous Apoptosis and Autophagy via Mitochondrial ROSMediated Akt-mTOR Signaling in Prostate Cancer Cells Expressing Mutant p53. Biomed Res. Int. 2013, 2013, 568671.

49. Jain, A., Lamark, T., Sjottem, E., Larsen, K. B., et al., p62/SQSTM1 is a target gene for transcription factor NRF2 and creates a positive feedback loop by inducing antioxidant response element-driven gene transcription. J. Biol. Chem. 2010, 285, 22576-22591.

50. Merendino, N., Loppi, B., D'Aquino, M., Molinari, R., et al., Docosahexaenoic acid induces apoptosis in the human $\mathrm{PaCa}-44$ pancreatic cancer cell line by active reduced glutathione extrusion and lipid peroxidation. Nutr. Cancer 2005, 52, 225233.

51. Natali, F., Siculella, L., Salvati, S., and Gnoni, G. V., Oleic acid is a potent inhibitor of fatty acid and cholesterol synthesis in C6 glioma cells. J. Lipid Res. 2007, 48, 19661975. 
52. Liu, Y., Zuckier, L. S., and Ghesani, N. V., Dominant uptake of fatty acid over glucose by prostate cells: a potential new diagnostic and therapeutic approach. Anticancer Res. 2010, 30, 369-374.

53. Hofer, C., Laubenbacher, C., Block, T., Breul, J., et al., Fluorine-18fluorodeoxyglucose positron emission tomography is useless for the detection of local recurrence after radical prostatectomy. Eur. Urol. 1999, 36, 31-35.

54. Altenberg, B., and Greulich, K. O., Genes of glycolysis are ubiquitously overexpressed in 24 cancer classes. Genomics 2004, 84, 1014-1020.

55. Vander Heiden, M. G., Locasale, J. W., Swanson, K. D., Sharfi, H., et al., Evidence for an alternative glycolytic pathway in rapidly proliferating cells. Science 2010, 329, 1492-1499.

56. Li, L., Fath, M. A., Scarbrough, P. M., Watson, W. H., and Spitz, D. R., Combined inhibition of glycolysis, the pentose cycle, and thioredoxin metabolism selectively increases cytotoxicity and oxidative stress in human breast and prostate cancer. Redox Biol. 2015, 4, 127-135.

57. Jump, D. B., Clarke, S. D., Thelen, A., and Liimatta, M., Coordinate regulation of glycolytic and lipogenic gene expression by polyunsaturated fatty acids. J. Lipid Res. 1994, 35, 1076-1084.

58. Clarke, S. D., Romsos, D. R., and Leveille, G. A., Differential effects of dietary methyl esters of long-chain saturated and polyunsaturated fatty acids on rat liver and adipose tissue lipogenesis. J. Nutr. 1977, 107, 1170-1181. 
59. Dentin, R., Benhamed, F., Pegorier, J. P., Foufelle, F., et al., Polyunsaturated fatty acids suppress glycolytic and lipogenic genes through the inhibition of ChREBP nuclear protein translocation. J. Clin. Invest. 2005, 115, 2843-2854.

60. Andrade-Vieira, R., Han, J. H., and Marignani, P. A., Omega-3 polyunsaturated fatty acid promotes the inhibition of glycolytic enzymes and mTOR signaling by regulating the tumor suppressor LKB1. Cancer Biol. Ther. 2013, 14, 1050-1058.

61. Valtonen-Andre, C., Bjartell, A., Hellsten, R., Lilja, H., et al., A highly conserved protein secreted by the prostate cancer cell line PC-3 is expressed in benign and malignant prostate tissue. Biol. Chem. 2007, 388, 289-295.

62.Pei, X., Sun, Q., Zhang, Y., Wang, P., et al., PC3-secreted microprotein is a novel chemoattractant protein and functions as a high-affinity ligand for CC chemokine receptor 2. J. Immunol. 2014, 192, 1878-1886.

63. Kue, P. F., Taub, J. S., Harrington, L. B., Polakiewicz, R. D., et al., Lysophosphatidic acid-regulated mitogenic ERK signaling in androgen-insensitive prostate cancer PC-3 cells. Int. J. Cancer 2002, 102, 572-579. 
Table 1 Differentially expressed proteins in cluster A and B. (*: t-test $\mathrm{p} \leq 0.05, * *$ : t-test $\mathrm{p} \leq 0.01, \mathrm{n}=5$ )

\begin{tabular}{|l|l|l|l|l|l|l|l|}
\hline Genes & Protein name & $\begin{array}{l}\text { Log2 } \\
\text { FO/CN } \\
\text { (day1) }\end{array}$ & $\begin{array}{l}\text { Log2 } \\
\text { OA/CN } \\
\text { (day1) }\end{array}$ & $\begin{array}{l}\text { Log2 } \\
\text { FO/CN } \\
\text { (day6) }\end{array}$ & $\begin{array}{l}\text { Log2 } \\
\text { OA/CN } \\
\text { (day6) }\end{array}$ & $\begin{array}{l}\text { ANOVA } \\
\text { p-value }\end{array}$ & q-value \\
\hline Cluster A & & & & & & & \\
\hline SCP2 & Non-specific lipid-transfer protein & -0.12 & -0.10 & $0.75^{* *}$ & $0.36^{* *}$ & $1.08 \mathrm{E}-04$ & $4.22 \mathrm{E}-03$ \\
\hline CD9 & CD9 antigen & 0.39 & -0.10 & $0.81^{* *}$ & 0.12 & $7.58 \mathrm{E}-05$ & $3.43 \mathrm{E}-03$ \\
\hline ACOX & Isoform 2 of Peroxisomal acyl-coenzyme A oxidase 1 & 0.25 & -0.29 & $0.52^{* *}$ & 0.14 & $8.71 \mathrm{E}-05$ & $3.80 \mathrm{E}-03$ \\
\hline ACOX1 & Acyl-coenzyme A oxidase & 0.30 & -0.28 & $0.55^{* *}$ & 0.12 & $7.29 \mathrm{E}-05$ & $3.43 \mathrm{E}-03$ \\
\hline SORD & Sorbitol dehydrogenase & 0.11 & -0.09 & $0.59^{* *}$ & 0.09 & $1.97 \mathrm{E}-07$ & $1.16 \mathrm{E}-04$ \\
\hline COTL1 & Coactosin-like protein & 0.11 & 0.05 & $0.31^{*}$ & 0.19 & $6.32 \mathrm{E}-05$ & $3.43 \mathrm{E}-03$ \\
\hline KPNA2 & Importin subunit alpha-2 & 0.08 & 0.02 & $0.29^{*}$ & 0.17 & $6.70 \mathrm{E}-04$ & $1.34 \mathrm{E}-02$ \\
\hline & & & & & & & \\
\hline Cluster B & & & & & & & \\
\hline APEH & Acylamino-acid-releasing enzyme (Fragment) & 0.11 & -0.07 & $-0.67^{*}$ & -0.28 & $5.33 \mathrm{E}-03$ & $4.94 \mathrm{E}-02$ \\
\hline TSTA3 & GDP-L-fucose synthase & $0.50^{*}$ & 0.12 & $-0.33^{*}$ & 0.12 & $1.05 \mathrm{E}-03$ & $1.85 \mathrm{E}-02$ \\
\hline SLC3A & Isoform 2 of 4F2 cell-surface antigen heavy chain & $0.59^{* *}$ & -0.02 & -0.26 & -0.18 & $1.20 \mathrm{E}-04$ & $4.44 \mathrm{E}-03$ \\
\hline SQSTM1 & Sequestosome-1 & $0.76^{* *}$ & -0.06 & 0.24 & 0.18 & $1.10 \mathrm{E}-06$ & $3.23 \mathrm{E}-04$ \\
\hline HSPA1A & Heat shock 70 kDa protein 1A/1B & $0.53^{* *}$ & -0.05 & -0.21 & 0.01 & $3.11 \mathrm{E}-03$ & $3.59 \mathrm{E}-02$ \\
\hline
\end{tabular}

Table 2. IPA pathway analysis of cluster $\mathrm{C}$.

\begin{tabular}{|l|l|l|}
\hline Molecular and Cellular Functions & & \\
\hline Name & p-value & Number of Molecules \\
\hline Cellular Growth and Proliferation & $6.61 \mathrm{E}-03-2.26 \mathrm{E}-08$ & 28 \\
\hline Cellular Movement & $6.61 \mathrm{E}-03-1.45 \mathrm{E}-06$ & 19 \\
\hline Cellular Development & $6.61 \mathrm{E}-03-2.10 \mathrm{E}-06$ & 23 \\
\hline Carbohydrate Metabolism & $6.13 \mathrm{E}-03-4.16 \mathrm{E}-06$ & 8 \\
\hline Cellular Death and Survival & $8.17 \mathrm{E}-03-4.42 \mathrm{E}-05$ & 22 \\
\hline & & \\
\hline Top Canonical Pathways & & \\
\hline Name & & Overlap \\
\hline Glycolysis I (GAPDH, GPI, PGK1, PKM) & p-value & $4 / 25$ (16\%) \\
\hline Gluconeogenesis I (GAPDH, GPI, PGK1) & $1.86 \mathrm{E}-07$ & $3 / 25(12 \%)$ \\
\hline Remodeling of Epithelial Adherens Junctions (TUBB3, TUBB4B, ZYX) & $1.78 \mathrm{E}-05$ & $3 / 68(4.4 \%)$ \\
\hline EIF2 Signaling (EIF3H, PABPC1, PPP1CB, RPL36) & $3.65 \mathrm{E}-04$ & $4 / 185$ (2.2\%) \\
\hline Breast Cancer Regulation by Stathmin1 (TUBB3, TUBB4B, PPP1CB, GNAI2) & $5.49 \mathrm{E}-04$ & $4 / 191(2.1 \%)$ \\
\hline
\end{tabular}


Table 3 Candidate targets of FO or OA treatment

\begin{tabular}{|c|c|c|c|c|c|c|c|c|c|}
\hline \begin{tabular}{|l|} 
Protein ID \\
\end{tabular} & Gene Name & Protein name & FC OA/CN & MP OA/CN & FCFO/CN & MPFO/CN & FC FO/OA & MP FO/OA & ANOVA q Value \\
\hline \multicolumn{10}{|l|}{ Day 1} \\
\hline E7EMC7 & SQSTM1 & Sequestosome-1 & -1 & 0.7 & 1.7 & 0.0001 & 1.8 & 0.0001 & 0.0003 \\
\hline \multicolumn{10}{|l|}{ Day 6} \\
\hline P22307 & SCP2 & Non-specific lipid-transfer protein & 1.3 & 0.005 & 1.7 & 0.0002 & 1.3 & 0.003 & 0.004 \\
\hline Q16658 & FSCN1 & Fascin & 1.1 & 0.08 & -1.2 & 0.05 & -1.4 & $6.00 \mathrm{E}-05$ & 0.009 \\
\hline B4DGP8 & CANX & Calnexin & 1.1 & 0.3 & -1.3 & 0.02 & -1.4 & 0.001 & 0.004 \\
\hline Q1L6U9 & MSMP & $\begin{array}{l}\text { Prostate-associated } \\
\text { microseminoprotein }\end{array}$ & -1 & & -2 & 0.0009 & -2 & 0.0008 & $2.12 \mathrm{E}-05$ \\
\hline
\end{tabular}

FC: fold change

MP: $p$ value of multiple comparison test 


\section{Figure Legends}

Figure 1: Effects of FO and OA on PCa cell viability. A: MTT assay was measured 24-hr after PC3 cells were treated by control ( $0.1 \%$ ethanol), $100 \mu \mathrm{M}$ fish oil or $100 \mu \mathrm{M}$ oleic acid $(n=3)$. B: PC3 cells were seeded at 1000 cell/well in 6-well plates, treated with control ( $0.1 \%$ ethanol), $100 \mu \mathrm{M}$ FO, or $100 \mu \mathrm{M}$ OA, respectively. Cells grew for approximately one week until the colonies were visible. Cells were fixed by methanol and stained with crystal violet $(n=3)$. C: PC3 cell images captured during colony formation.

Figure 2: Effects of FO and OA on FASN activity in PC3 cells. Cells were seeded in 24-well cluster plates, treated with vehicle (0.1\% ethanol), $100 \mu \mathrm{M}$ FO or $100 \mu \mathrm{M} \mathrm{OA}$ for 3 days or 6 days. FASN activity was measured by ${ }^{14} \mathrm{C}$-acetate incorporation assay, in which the scintillation counts were normalized by cell counts in parallel treated wells $(n=3)$.

Figure 3: Principal component analysis (PCA). A: PCA analysis of 127 significantly changed proteins (ANOVA $p \leq 0.05, q \leq 0.05, n=5$ ) shows graphic distribution of 30 samples (6 groups and 5 replicates in each) on axes of component 1 (F1) and component 2 (F2). B: By one minus Pearson Correlation, three major clusters were generated out of 73 proteins selected based on 127 proteins in $(A)$ and has at least one significant change (t-test $p \leq 0.05, n=5$ ) in FO1/CN1 (fish oil vs. control, one-day), OA1/CN1 (oleic acid vs. control, one-day), FO6/CN6 (fish oil vs. control, six-day), or OA6/CN6 (oleic acid vs. control, six-day). 
Figure 4. Enzyme functions in glycolysis/gluconeogenesis and its relative pathways are changed. Differentially expressed glycolysis/gluconeogenesis proteins in cluster C (*: ttest $p \leq 0.05, n=5)$

Figure 5: MRM validation of expression changes of MSMP (A), SCP2 (B) in FO or OA group compared to control, with internal control RPL6 (C) and spiked-in external control chicken lysozyme (D) $(n=6)$.

Figure 6: Western Blot validation of expression changes of MSMP, SQSTM1 and autophagy marker LC3 I/II in FO or OA group. (A) SQSTM1 and $\beta$-actin; (B) Bar graph quantification of (A), n=3; (C) SQSTM-1 mRNA level by RT-PCR (GUSB mRNA serves as internal standard), $n=5$; (D) Western blot of LC3 I/II from cells treated with different FAs in the presence of $8 \mu \mathrm{M} \mathrm{CQ}$ for 1 day; $(E)$ Bar graph quantification of $(D)$, $\mathrm{n}=3$; $(\mathbf{F})$ secreted MSMP in medium; (G) Bar graph quantification of $(\mathrm{F}), \mathrm{n}=3$. 